\title{
Metal-regulated transcription in eukaryotes
}

\author{
Dennis J.Thiele \\ Department of Biological Chemistry, University of Michigan Medical School, Ann Arbor, \\ MI 48109-0606, USA
}

Received November 14, 1991; Revised and Accepted February 5, 1992

\section{INTRODUCTION}

The major role metals play in biology is highlighted by the fact that metals compose 17 of the 30 elements known to be essential for life (1). Metals constitute a nutritionally important, yet cytotoxic component in our environment (2). A number of metals can be categorized into three groups with respect to these considerations. One group, of which zinc $(\mathrm{Zn})$ is a good example, provides essential cofactors for a wide variety of metalloproteins and enzymes. This metal is normally not toxic except at extremely high concentrations. A second group, of which cadmium (Cd) is a member, constitutes metals which have no known nutritional value yet are highly cytotoxic. The third group is composed of metals such as copper $(\mathrm{Cu})$, which are both essential for life processes as cofactors for many enzymes and are extremely potent cellular toxins (2). Indeed, because metal concentrations in our environment change in response to diet, habitat and environmental pollutants, organisms must constantly assess environmental metal concentrations and respond in physiologically meaningful ways. To date, one of the clearest examples of physiological responses to changing environmental metal concentrations is manifested in the activation or repression of gene transcription. Metal-regulated gene transcription has been well documented in a wide variety of organisms including bacteria, fungi, insects, plants, fish, rodents and humans. A great deal of information is known about the occurrence and biochemical mechanisms for metal-regulated transcription in prokaryotic cells and the reader is referred to a recent comprehensive review of this area by O'Halloran (3). Although metal responsive gene transcription in eukaryotic organisms has been intensively investigated for many years, only recently have the biochemical mechanisms for this process begun to be understood. In this article, I will review the current status of our understanding of the mechanisms by which eukaryotic organisms sense changes in environmental metal levels and respond to this sensation by regulating the transcription of specific cellular target genes.

Metallothioneins as model metal-regulated transcription units In higher eukaryotes, the metallothionein (MT) genes have been the most intensively studied and best understood examples of metal-regulated transcription units. MT genes encode low molecular weight, cysteine-rich metal binding proteins found in a wide range of eukaryotes and are thought to play several important roles in metal homeostasis (4). The well established role for MT proteins in protection from metal toxicity correlates with the ability of several metals, including zinc, copper, cadmium, lead and others, to rapidly and potently activate MT gene transcription (5). Work in Palmiter's laboratory on the isolation and analysis of the mouse metallothionein I (mMT-I) cDNA and gene provided the first experimental tools for establishing the metal-activated transcription of MT genes (6). I will focus this section on metal-activated transcription of higher eukaryotic MT genes. Furthermore, I will use as a framework for this discussion, the analyses of the mouse metallothionein I promoter, since this is the most thoroughly studied of the higher eukaryotic metal-regulated transcription systems.

Deletion and linker-scanner mutations constructed in the mouse MT-I promoter led to the initial conclusion that multiple elements were present which contribute to the overall magnitude of metalresponsive transcription $(7,8)$. These cis-acting MREs (Metal Responsive Elements), present in multiple copies in all MT promoters analyzed to date, are composed of a series of 13-15 bp imperfect repeats and are found in both orientations within MT promoters (9-13) (Fig.1). A series of comprehensive point mutagenesis studies, combined with MRE sequence comparison analyses, established the presence of an MRE core sequence which is vital for efficient metal-inducible transcription $(14,15)$. The consensus MRE element core sequence, shown in Fig. 2., bears no obvious resemblance to the binding sites for ACE1 or AMT1, the copper-activated Metalloregulatory Transcription Factors (MRTFs) from the yeasts Saccharomyces cerevisiae and Candida glabrata, respectively (see below).

Several features of MREs are noteworthy with respect to their mechanism of action. In studies using synthetic copies of the mMTI MREs, Stuart et al. have demonstrated that the individual MRE elements vary in their metal-activated transcriptional potency when placed in front of the Herpes Simplex Virus tk (thymidine kinase) promoter (16). The mouse MT-I promoter harbors 6 MREs designated MREa through MREf. Dimers of mMREd foster a 20 -fold induction in response to zinc in this context, indicating that mMREd is the strongest of the mouse MT-I promoter MREs. Westin and Schaffner have demonstrated that a multimerized MREd has properties of a metal-inducible enhancer, since it can activate the rabbit $\beta$-globin gene promoter in HeLa cells over a long distance, in either orientation and either upstream or downstream of the $\beta$-globin transcription initiation site (17). Furthermore, the mMT-I, human $\mathrm{MT}^{\mathrm{T}}-\mathrm{II}_{\mathrm{A}}$ and several other MT promoters have MREs interdigitated with other regulatory elements $(7-13)$, suggesting the possibility for a number of functionally significant protein-protein interactions and other regulatory mechanisms. Finally, it has been clearly demonstrated that a single synthetic MRE (mMREd) is transcriptionally responsive to the same set of metals known to induce transcription of the authentic mMT-I promoter $(14,15)$. 
The mouse MT-I gene MREs are the most intensively studied and best characterized of the higher eukaryotic metal-responsive promoter elements. Since a single MRE is transcriptionally responsive to multiple distinct metals, how do MREs mediate transcriptional activation of MT and other metal-responsive genes? The underlying model proposed by many laboratories postulates the existance of one or more metal-responsive transcription factors, perhaps similar in nature to the two characterized fungal MRTFs described later in this review. Implicit in this model is the presence of a pre-existing metal sensory factor, a feature supported by experiments which demonstrate the lack of a requirement for de novo protein synthesis for MT gene activation by metals (18). A number of compelling experimental results support this model for metal-activated transcription of higher eukaryotic MT genes. Using in vivo competition experiments with a mouse MT-I galK fusion gene as indicator, and wild type or mutated MREs on mMT-I promoter derivatives as competitor, the existance of a titratable positively-acting metal-responsive transcription factor was demonstrated (19). Using more direct approaches, two laboratories subsequently demonstrated in vivo metal-dependent DNA-protein interactions between MREs and one or more cellular factors. Employing dimethylsulfate (DMS) to assess guanosine methylation in vivo as a function of MT promoter activation, Herschman and colleagues detected clear cadmium-dependent DNA-protein interactions at all five MREs present in the rat MT-I gene promoter (20). Combining both DNase I and DMS footprinting in vivo on L cells containing multiple copies of an amplified mouse MT-IDHFR fusion gene, Wold's laboratory carried out a detailed analysis of MT-I promoter-protein interactions (21). These investigators detected zinc-dependent protein-DNA interactions at all five MREs $(a-e)$ previously characterized genetically and furthermore, obtained evidence for a sixth MRE (MREf) located between MREs b and c. Interestingly, MREf is less efficiently protected from DMS methylation in L cells treated with cadmium than zinc, although all other MREs were affected identically by these metals. The significance of the different response of MREf remains unclear. Furthermore, these studies demonstrated that MREd, the most potent metal-responsive element in vivo, is occupied in vivo in cells not exposed to exogenous zinc. Although MREd is interdigitated with a binding site for the transcription factor Sp1 (17), the pattern of occupation observed in these in vivo assays is not consistent with the binding of Spl exclusively. These observations suggest that either the MREd region serves as a target site for several DNA binding proteins in vivo, or that, consistent with the activation potency of this element in vivo, this represents a very high affinity binding site which can be occupied by a low level of active MRTF in these cells. These in vivo footprinting studies just described $(20,21)$ set the stage for in vitro analyses.

In vitro evidence for a metal-dependent MRE-binding protein has, until recently, been elusive. Several reports have

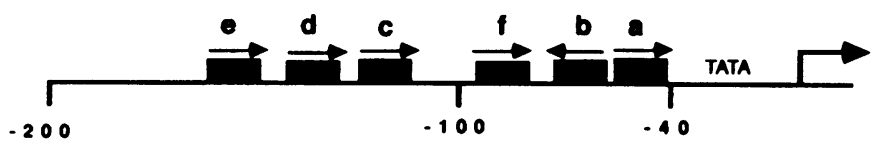

Figure 1. Organization of the Metal Responsive Elements (MREs) within the mouse metallothionein I (mMT-I) promoter. The location and orientation of the mMREs a through $f$ is indicated relative to the binding site for Transcription Factor IID (TATA) and the start site for transcription (arrow). This diagram summarizes data obtained both by functional analysis and in vivo DNA binding studies $(7,8,21)$. demonstrated the existence of metal-dependent DNA binding activities from both tissue culture cells and whole tissues. Interestingly however, each report of such an activity has demonstrated metal-activated MRE-binding in response to only a single metal, even though as described above, a single mouse MRE is responsive to all metals known to activate mouse MT-I transcription $(14,15)$. Consistent with in vivo DMS footprinting of the rat MT-I promoter, Andersen et al. (22) observed the formation of a Cd-inducible DNA-protein complex, of relatively low affinity, using either a large promoter fragment encompassing multiple MREs or a fragment encompassing the rat MREs $\mathrm{c}$ and d. Indeed, complex formation was only observed with $\mathrm{Cd}$ and no other metals and was too weak to detect protein binding by standard footprinting procedures. Furthermore, the binding of this factor required at least two MREs. Crosslinking experiments identified an approximately $39 \mathrm{kd}$ polypeptide as part of this $\mathrm{Cd}$ inducible DNA-protein complex.

Although in higher eukaryotic systems $\mathrm{Cd}$ appears to be the most potent metal for MT gene induction (5), many investigators have focused on the detection of $\mathrm{Zn}$-responsive MRE-binding factors, an approach which is complicated by the fact that many MREs lie juxtaposed to binding sites for other transcription factors, of which some are known to be zinc-finger-type DNA binding proteins such as Spl (23). Westin and Schaffner demonstrated that the mMT-I MREd site contains both a functional Spl binding site and serves as a binding site for a zincactivated (but not Cd-responsive) factor from HeLa cells (17). Methylation interference analyses and the use of functionally impaired MREs in binding assays suggest that Sp1 and the $\mathrm{Zn}$ dependent factor, denoted MTF-1 (Metal Dependent Transcription Factor), have overlapping but non-identical DNA binding specificities. In view of the overlap between mouse MT-I gene MREs and other cis-acting regulatory elements, Imbert $e t$ al. (24) used an affinity chromatography based method to purify a mouse protein which binds an oligonucleotide containing an MRE element from the trout MT-B gene (tMRE). These experiments were based on the absence of experimental evidence for overlapping regulatory elements with the tMRE and the observation that a trout MT-B gene promoter-CAT fusion, harboring only a functional tMREa element, was able to direct modest $\mathrm{Cd}$ and $\mathrm{Zn}$ activated transcription in mouse $\mathrm{L}$ cells as determined by transfection experiments. These investigators affinity-purified to near homogeneity a mouse factor designated MBF-I (Metal Response Element Binding Factor I), of approximately $74 \mathrm{kd}$, which bound an oligonucleotide containing a functional MRE, but not a non-functional oligonucleotide with point mutations in critical MRE core residues. A number of aspects of MBF-I remain to be resolved with respect to its role

\section{MRE consensus CTCTGCRCNCGGCCC}

\section{ACE1 binding site TCY(4-6)GCTG}

Figure 2. Comparison of the sequence of the consensus Metal Responsive Element (MRE) from higher eukaryotic MT genes with the ACE1 consensus binding site in $S$. cerevisiae. Bolded residues in the MRE consensus are essential for the functional integrity of the MRE as a metal-responsive cis-acting element as determined by transfection experiments $(14,15)$. Bold type residues in the $S$. cerevisiae ACE1 consensus binding site are important for ACEl binding, as determined by methylation interference assays $(39,45,56,60,76)$. The ACEl consensus is drawn from binding sites in the $S$. cerevisiae $C U P I$ and $S O D I$ genes. $\mathrm{R}=$ purine, $\mathrm{Y}=$ pyrimidine. 
in vivo. First, MBF-I binding to tMRE is not stimulated in vitro by zinc, and although tMRE-driven transcription in mouse $\mathrm{L}$ cells is stimulated by both $\mathrm{Cd}$ and $\mathrm{Zn}$, in vitro MBF-I modestly stimulates transcription from a tMRE-containing template only in the presence of zinc. Furthermore, although MBF-I was purified from mouse $\mathrm{L}$ cells, there has been no experimental evidence presented which demonstrates MBF-I binds to mouse MTI functional MREs in vitro. Recently, using sequence information derived from an MBF-I preparation, a cDNA was isolated which corresponds to the RP-A factor, a zinc finger protein which functions in DNA replication (25) (J. Imbert and D. Hamer, personal communication). The relationship of this clone to MBF-I activity and metal activated transcription of the mMT-I gene remains to be elucidated.

Several recent reports from Seguin $(26,27)$ and the laboratories of P. Searle (28) and W. Schaffner (17) strongly suggest the presence of a specific zinc-activated MRE binding protein found in both mouse L cells, rat liver nuclear extract, and HeLa cells. In vitro footprinting experiments of Seguin and co-workers have demonstrated the presence of a mMT-I MREd binding activity which is inactivated by the metal chelating reagent 1,10-phenanthroline, and that, once inactivated, binding can be restored by zinc addition but by no other metal tested (26). Competition studies in vitro have demonstrated that other transcriptionally competent MREs, but not those bearing nonfunctional point mutations, could compete for the binding to MREd. Furthermore, the competitive strength of the mouse MRE oligonucleotides corresponded well to the respective in vivo transcriptional potency. These results clearly demonstrate that the Zn-dependent DNA binding factor, denoted MEP-1 (Metal Element Binding Protein) binds to all mMT-I MREs and in a fashion consistent with a function as a metal-activated transcription factor in vivo. A Southwestern analysis (protein blotting procedure) has demonstrated that MEP-1 activity copurifies with a polypeptide of $108 \mathrm{kd}$ (27), which has been purified to homogeneity as an apparently unglycosylated protein which adheres to a zinc chelating sepharose column (C. Seguin, personal communcation). Interestingly, P. Searle has demonstrated the existence of a rat liver nuclear protein which binds to the mMT-I MREa in a zinc-dependent manner, as demonstrated by electrophoretic mobility shift assays (28). The demonstration of this activity, termed ZAP (Zinc Activated Protein), is important for several reasons: First, MREa is capable of metal-regulation in vivo without apparent overlapping regulatory sites. Secondly, ZAP binding depends on a transcriptionally competent MRE core element. Furthermore, ZAP binding in vitro is maximally activated by $60-100 \mu \mathrm{M}$ $\mathrm{ZnSO}_{4}$, the same concentration range known to efficiently induce mMT-I transcription in tissue culture (6). As for MEP-1, neither $\mathrm{Cd}$ nor $\mathrm{Cu}$ stimulated ZAP binding to MREa. Similarly, Schaffner's laboratory has demonstrated the presence of a zincinducible mMT-I MREd binding activity, termed MTF-1, in HeLa cell nuclear extracts (17). Since ZAP also binds mMREd in a zinc-dependent manner, it is possible that ZAP and MTF-1 possess similar activities. Furthermore, prelimary Southwestern blot assays suggest that ZAP is in the same size range as MEP-1, perhaps indicating that MEP-1, ZAP and MTF-1 have analogous functions in metal-regulation of MT genes in different organisms. Indeed, the demonstration that MREs from Drosophila foster metal activated transcription in mouse cells suggests a very high level of conservation of the DNA binding specificity of MRTF proteins from higher eukaryotes (10). Although insight into the role MEP-1, MTF1 and ZAP play in mMT-I gene transcription awaits analyses by in vitro transcription reactions, the ability to purify these factors represents a major step toward cloning the corresponding cDNA. Since an in vitro transcription system for the mouse MT-I gene has already been established (24), functional analyses of these factors should be greatly facilitated.

The experimental evidence published to date suggest the presence of multiple MRE binding factors with metal-dependent activities. Based upon previous demonstrations that a single MRE is responsive to many metals known to induce MT gene transcription, how can these observations be reconciled? Although cDNAs encoding metal-responsive trans-activators must first be isolated and analyzed to clarify the mechanisms for metal regulated MT gene transcription, we can invoke a number of models to explain the observations. First, given the abundance of families of structurally-related transcription factors in higher eukaryotes (see for example 30), it is possible that multiple MRTF genes exist which encode proteins with distinct metal specificities. The known differences in preferred coordination chemistry between metals such as $\mathrm{Zn}$ (tetrahedonal) and $\mathrm{Cu}$ (trigonal) would be consistent with this possibility (1), however, this would not explain the inability of activities such as MEP-1, MTF-1 or ZAP to respond to $\mathrm{Cd}$, a metal stably tetrahedonally coordinated much like zinc (1). Should the MRTF gene family model be operational, however, it would require that the metal-activated MRTFs all recognize and bind to the critical MRE core sequence in vivo. A second model for the mechanism of action of higher eukaryotic MRTFs I refer to as the flexible protein model. This scheme postulates that a single promiscuous MRTF protein exists in higher eukaryotic cells which can sense and respond to a variety of different metals. Again, given the predicted structural differences which would result from metal-specific preferred coordination geometries, such a protein must assume a DNA binding domain structure which would allow specific interactions with MREs regardless of the metal bound. Although other, less direct mechanisms of metal-regulated transcription may operate in higher eukaryotes, experimental observations so far are consistent with the existence of one or more metal-activated MRE-binding transcription factors. Consistent with the possibility that nuclear metal activated DNA binding proteins bind to and activate transcription from MREs, Cousins and co-workers have shown that dietary zinc is rapidly taken up by rat kidney nuclei (31). Furthermore, these investigators demonstrated that a chromatographically separated nuclear zinc (as ${ }^{65} \mathrm{Zn}$ ) binding activity binds a highly conserved trout MRE oligonucleotide (31). These studies underscore the importance of dietary metal sources, and the transport of metals into cell nuclei, as important aspects of any studies aimed at understanding metal-regulated gene transcription in eukaryotes.

\section{Yeast Metallothionein Genes as a Model Eukaryotic Metallo- regulatory System}

Lower eukaryotes have provided a great deal of insight into a large number of biological regulatory systems due to their ease of genetic and biochemical manipulation, their amenability to a vast array of molecular techniques of analysis and the ability to precisely control the growth conditions of these organisms. Indeed, studies of metal-regulated gene transcription in yeast cells have provided the first information about the structure and mechanism of action of eukaryotic metalloregulatory transcription factors (MRTFs). In this section, I will summarize the current 
body of knowledge of metal-regulated gene transcription in two yeasts, the baker's yeast Saccharomyces cerevisiae and the opportunistic pathogenic yeast Candida glabrata.

\section{Saccharomyces cerevisiae}

The metallothionein gene in S. cerevisiae, designated CUP1, has been the principal system for studies of metal-regulated gene transcription in this organism. For the purpose of this review, it is important to know that the CUPI locus was originally identified as a dominant Mendelian locus which confers copper resistance upon yeast cells $(32,33)$. Subsequent chromosomal deletion studies, carried out by homologous recombination, clearly demonstrated that $C U P 1$ is a major determinant in copper resistance in $S$. cerevisiae $(34,35)$. This gene encodes a 61 amino acid MT protein which protects cells by binding intracellular copper, thereby preventing the toxic effects of this metal $(36,37)$. Biochemical experiments have demonstrated that the CUPI protein binds 8 copper molecules per polypeptide, in a trigonal coordination array, through cysteine thiolates in a $\mathrm{Cu}-\mathrm{S}$ polynuclear cluster (38). The implications of this mode of metal binding with respect to the fungal metalloregulatory transcription factors will be discussed below.

Initial investigations of CUPI transcription demonstrated that, in contrast to the wide array of metals which induce mammalian MT gene transcription, copper was the only metal capable of inducing CUP1 transcription (36,37). Subsequent studies further demonstrated that silver $(\mathrm{Ag})$ is also an effective, although less potent, inducer of CUP1 transcription (39). Whether this decreased effectiveness is due to the extreme toxicity of $\mathrm{Ag}$, to differences in its uptake or interaction with the metalloregulatory factor, or to multiple causes, have not yet been firmly established. Interestingly, cadmium has been shown to either slightly induce or repress CUP1 transcription $(36,40)$, however whether these are direct or indirect effects on CUP1 transcription have not been resolved. The major point for both $C U P 1$ transcription and MT gene transcription in other lower eukaryotes (41) is the high degree of metal specificity as compared to the MT genes of higher eukaryotes.

The cis-acting promoter elements important for copperinducible transcription of $C U P 1$ have been investigated by both promoter deletion and point mutation analysis. Initially, a series of $3^{\prime}, 5^{\prime}$ - and internal CUPI promoter deletions were assayed for their ability to drive copper-inducible transcription of a CUPI promoter-E. coli galactokinase (galK) fusion gene in vivo (42). These experiments revealed the presence of multiple regions of the $C U P 1$ promoter which are important for copper-inducible transcription, localized between approximately -105 and -230 with respect to the transcription initiation site. This region has been designated the CUPI Upstream Activation Sequence (UAS $\mathrm{CUP1}_{\mathrm{C} 1}$ ). A synthetic oligonucleotide representing sequences between -108 and -139 , when inserted into a CYCl-galk minimal promoter fusion in tandem, was shown to activate transcription of this heterologous promoter in response to copper, although an order of magnitude less well than the intact CUPI promoter (42). A single such oligonucleotide gave rise to only minor copper-inducible transcription in this assay system. Furthermore, the orientation of insertion of oligonucleotide was critical to its ability to drive copper-inducible transcription of the CYC1-galK reporter gene. These results supported the hypothesis for a positively acting metal-responsive transcription factor.

The important role that $C U P I$ plays in copper resistance in $S$. cerevisiae was critical to classical genetic studies which identified the first eukaryotic metalloregulatory transcription factor. Based on the observations that $C U P 1$ is necessary for resistance to high levels of copper, and that CUPI is transcriptionally activated by this same metal, the presence of a copper-regulated transcriptional regulatory factor was postulated and sought by genetic means. Using the general mutagen ethyl methanesulfonate (EMS), two laboratories independently isolated copper sensitive strains from otherwise highly resistant parental strains, which failed to activate CUPI transcription in trans in response to elevated environmental copper levels $(43,44)$. These recessive mutations, designated acel-1 (activation of CUPI expression) and cup2, were subsequently demonstrated to be allelic (45), therefore, for simplicity in this review, the designation $A C E 1$ will be used throughout to refer to both the wild-type $A C E 1 / C U P 2$ genes and their identical gene products. The wild-type ACE1 gene was cloned by transforming a strain bearing the ace 1-1 mutant allele and selecting for copper-resistant recipients due to the restoration of copper inducible CUPI transcription (43).

Several features of ace 1-1 mutants and the cloned ACE1 gene provided initial insight into the mechanism by which ACE1 activates CUP1 transcription in trans. Welch et al. (44) demonstrated that cells harboring a defective ACE1 gene lack $\mathrm{UAS}_{\text {CUPI }}$ binding activity which is present in wild type cells. Furthermore, it was demonstrated that ACE1 gene expression is unaffected by copper either at the level of transcription or translation (46). Consistent with ACE1 acting as a direct transcription factor is the demonstration that a transcriptionally competent $\mathrm{ACE} 1-\beta$-galactosidase fusion protein concentrates in the yeast cell nucleus (46). In addition, deletion of either the 3 '-half or the entire $A C E 1$ gene demonstrated that $A C E 1$ is not essential for viability under standard laboratory conditions $(43,47)$. Furthermore, strains bearing deletion alleles of ACE1 exhibit lower levels of CUPI transcription in the absence of exogenous copper, indicating that ACE1 plays a role in both basal level and copper-induced CUPI transcription (47). As I will describe in detail in a later section of this review, ACE1 is a copper-activated DNA binding transcription factor that directly interacts with UAS ${ }_{\mathrm{CUP1}}$. Its mode of binding and the interesting structural features of ACE1, and a second MRTF, AMT1 from Candida glabrata, are discussed below.

\section{Candida glabrata}

The opportunistic pathogenic yeast Candida glabrata has provided an interesting and more complex fungal metalloregulatory system than baker's yeast. Through a number of informative studies, Winge's laboratory demonstrated that, like higher eukaryotes, C. glabrata contains a family of metallothionein genes: a unique MT-I gene, a tandemly amplified MT- $\Pi_{\mathrm{a}}$ gene and an apparently single-copy $\mathrm{MT}$ - $\mathrm{II}_{\mathrm{b}}$ gene $(48,49)$. In agreement with the biosynthetic regulatory features of the $S$. cerevisiae CUPl gene, however, the $C$. glabrata MT genes are transcriptionally activated only by the metals $\mathrm{Cu}$ and $\mathrm{Ag}$ (48). Therefore, C. glabrata exhibits the multiplicity of distinct MT genes typical of higher eukaryotes but a metal specificity for MT biosynthetic regulation similar to that found in $S$. cerevisiae, providing an interesting model system to examine metal-regulated transcription. Due to the lack of existing genetics with this organism, surrogate genetic experiments in $S$. cerevisiae were conducted to clone a $C$. glabrata MT metalloregulatory factor gene (50). An $S$. cerevisiae strain was constructed which lacks both the CUPI and $A C E 1$ genes, thereby rendering these cells 
exquisitely sensitive to copper poisoning. At two unlinked chromosomal loci, a single copy of the $C$. glabrata MT-I gene and promoter region, and an $M T-I-l a c Z$ fusion, were integrated to allow the detection of $C$. glabrata copper-dependent transactivator proteins in this host strain transformed with a $C$. glabrata genomic DNA library on an $S$. cerevisiae multicopy vector. A single positive transformant was obtained which rendered the recipient colonies both copper-resistant and blue on $\mathrm{X}$-gal (the chromogenic substrate for $\beta$-galactosidase) indicator media. The functional $C$. glabrata DNA fragment, denoted AMT1 (Activator of Metallothionien Transcription 1) was shown to encode a 265 amino acid polypeptide with regions of striking primary structural similarity to the ACE1 protein from $S$. cerevisiae (see below).

Two published experimental observations suggest that AMT1 activates $M T-I$ and $M T-I I$ gene transcription in vivo. First, is the ability of the AMT1 protein to act in concert with the basic transcriptional machinery of $S$. cerevisiae for trans-activation (50). Second, soluble AMT1 protein produced in $E$. coli gives rise to multiple DNA-protein complexes, in a copper-or silverdependent manner, with both the $M T-I$ and $M T-I I_{\text {a }}$ gene upstream promoter fragments (50). Recent results have firmly established the in vivo role of AMT1 as the positive metalloregulatory factor for the $C$. glabrata $M T-I$ and $M T-I I_{\mathrm{a}}$ genes. Chromosomal disruption of the $A M T 1$ gene renders $C$. glabrata cells both extremely copper-sensitive and unable to induce $M T-I$ or $M T-I I_{\mathrm{a}}$ mRNA levels in response to elevated environmental copper levels (Zhou, et al. submitted). Although it has been proposed that MT gene copy number in $C$. glabrata does not strictly correlate with copper resistance levels (51), it is clear that the expression of MT genes in response to metalactivated $A M T 1$ is of critical importance in the copper detoxification pathway.

\section{Structure-function relationships in fungal metalloregulatory factors}

A major question concerning metalloregulatory transcription factors and their structure-function relationships is: how do these proteins sense elevated environmental metal concentrations and transmit this sensation to the transcriptional machinery? As I describe below, the simple answer to this question is that eukaryotic MRTFs known to date directly bind metals and, as a result, are activated to bind specific promoter DNA sequences to activate transcription. Currently, however, the nature of the tertiary structure of metallated MRTFs and the mechanisms by which, once bound to DNA, they activate transcription, are areas of intense investigation. Furthermore, the structural or functional complexity of other eukaryotic MRTFs, which may respond to many metals or which may activate or repress gene transcription, is currently a poorly understood area of investigation.

First, how do eukaryotic MRTFs bind metals? The two eukaryotic MRTF genes isolated to date, $A C E 1$ (CUP2) from $S$. cerevisiae and $A M T 1$ from the evolutionarily related (52) $C$. glabrata have provided indispensable tools for understanding the answers to this question, at least for fungal MRTFs. $A C E 1$ protein has a remarkable distribution of amino acid residues throughout the 225 aa polypeptide chain (46). Twelve cysteine residues are present within the amino terminal 105 aa, with this same region bearing a net charge of +16 . In contrast, the carboxyl-terminal 120 aa. residues of $\mathrm{ACE} 1$ have a net charge of -14 , a clear assymetric distribution of charge in this MRTF. The presence of the 12 Cys residues within a region of high positive charge suggested this portion of ACE1 constituted the copper-activated DNA binding domain. Secondly, the arrangement of the Cys residues in Cys-X-Cys or Cys- $\mathrm{X}_{2}-\mathrm{Cys}$ configurations, known to be important for metal coordination in MTs and other metalloproteins, suggested that these represent ligation sites for copper (38). In vitro mutagenesis and DNA binding studies with ACE1 support both conclusions. The production of the amino-terminal 105 aa of ACE1 either by in vitro transcription-translation or in $E$. coli demonstrated that this region encompasses the copperactivated DNA binding domain $(39,53)$. The analysis of ACE1 bearing point mutations generated either in vitro or in vivo indicates that the amino terminal 11 Cys residues are, individually, of critical importance for the copper-activated DNA binding function $(43,45,53)$. These observations suggest that the $\mathrm{Cu}-\mathrm{ACE} 1$, by virtue of the eleven critical Cys residues, forms a higher order structure essential for transcriptionally competent DNA binding. Importantly, a 115 aa amino-terminal portion of the $C$. glabrata AMT1 protein, which retains copper-activated DNA binding capacity indistinguishable from authentic AMT1, also harbors 11 Cys residues, (50, Zhou et al., submitted). The first seven Cys residues in ACE1 and AMT1 are precisely conserved in their position and arrangements within the amino-termini, with the next 4 Cys residues in each protein also alignable if the 10 additional AMT1 residues in this region are considered (50).

Initial atomic absorption studies of copper-saturated wild-type $\mathrm{ACE} 1$, and acel-1 protein harboring a Cys ${ }^{1} \rightarrow \mathrm{Tyr}$ change, demonstrated that the mutant protein has two noteable properties. Consistent with the proposed role of the Cys residues in $\mathrm{Cu}$ coordination, wild type ACE1 was shown to bind $\mathrm{Cu}$ in a 1:6 molar stoichiometry, whereas the ace 1-1 protein bound in a 1:5 stoichiometry. Secondly, this ace 1-1 derivative defective in $\mathrm{Cu}$ binding and in vivo trans-activation was shown to interact with

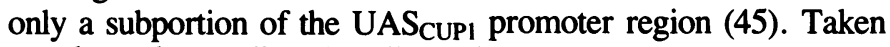
together, the studies described above suggested that the Cys residues in ACE1 are important for $\mathrm{Cu}$-coordination and conferring an active DNA binding structure on $\mathrm{Cu}-\mathrm{ACE} 1$. Indeed, partial proteolysis studies suggest that $\mathrm{Cu}-\mathrm{ACE} 1$ adopts a protease resistant structure in its amino-terminus which is distinct from the apoprotein (39).

Recently, biochemical and biophysical studies of the ACE1 DNA binding domain have begun to yield important information as to the mode of copper binding. Extended X-ray absorption fine structure (EXAFS) analysis of authentic ACE1 made in $E$. coli clearly demonstrated that the $\mathrm{Cu}$ is sulfur coordinated in the $\mathrm{Cu}(\mathrm{I})$ oxidation state with a three-fold coordination geometry (54). The $\mathrm{Cu}$-sulfur distance of $2.26 \AA$ is consistent with that observed for model trigonal coordination compounds and suggests that the $S$ groups are contributed by the critical Cys residues within the ACE1 DNA binding domain. Similar results were obtained using an ACE1-bacteriophage $\mathrm{T} 7$ gene 10 fusion protein purified from $E$. coli (55). Furthermore, the observation of $\mathrm{Cu}-\mathrm{Cu}$ interactions indicate that ACE1 forms a Cu-cluster, which is consistent with the inferred cooperativity in copper binding based on copperactivated DNA binding and protease resistance assays (56). Although Ag activates CUPI transcription in vivo and ACE1 DNA binding to UAS $\mathrm{UUPI}_{\mathrm{CU}}$ in vitro (39), the reduced efficiency for both of these functions suggest that Ag-ACE1 has a lower affinity for $\mathrm{UAS}_{\mathrm{CUP1}}$ and may not allow ACE1 to assume precisely the same DNA binding structure as does $\mathrm{Cu}$. Nonetheless, it has been suggested that the trigonal coordination of copper by ACE1 protein confers the metal specificity on this transcription factor (54). The observation that ACE1 and AMT1 from C. glabrata have similar 
primary structures and respond to the same metals for both in vivo trans-activation and in vitro DNA binding suggest that these proteins may bind copper and DNA by very similar mechanisms. Understanding the three-dimensional structures of $\mathrm{Cu}-\mathrm{ACE} 1$ and $\mathrm{Cu}$-AMT1 should ultimately provide a great deal of information for a comprehensive understanding of how MRTFs sense metals and rapidly transmit this information to the transcriptional machinery. It is also interesting to note that the yeast MT negatively autoregulates its own transcription. CUP1 promoter fusions, when present in CUP1 deletion (cupl $\Delta$ ) strains, have constitutively high basal levels of expression (34), which can be returned to normal low levels by introduction of either the wild type CUP1 gene or expression of either of two monkey MT isoforms (57). Furthermore, copper-binding forms of yeast MT are required to re-establish low basal levels of transcription (58). These results suggest that yeast MT negatively autoregulates its transcription by basal protein levels binding to intracellular free copper which would otherwise be available for ACE1. When CUPI is deleted, physiological levels of available copper are elevated, thus activating

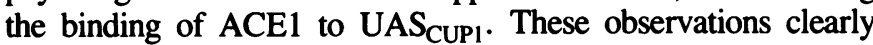
demonstrate the necessity for understanding metal homeostasis as an integral part of metal regulated gene transcription, and reinforce the fact that gene transcription cannot always be studied independently of gene product function.

How do fungal MRTFs bind their cognate promoter elements in vivo? Using a chromosomal footprinting approach, Huibregtse et al (59) demonstrated that ACE1 protein binds three distinct regions within $\mathrm{UAS}_{\mathrm{CUP1}}$ in a copper-inducible manner. Subsequent work demonstrated that ACE1 binds to these same three regions, comprising four independent binding sites, as a monomer in vitro $(56,60)$. Therefore, consistent with the presence of multiple MRE sequences in higher eukaryotic MT genes, ACE1 binds multiple sites in the CUPI promoter. Since yeast genomic DNA is organized into nucleosomes, which have been shown to modulate transcription (61), is there an effect of chromatin structure on ACE1 binding to $\mathrm{UAS}_{\mathrm{CUP1}}$ ? Although data regarding nucleosome structure within $\mathrm{UAS}_{\mathrm{CUPI}}$ has not been reported, recently Grunstein's laboratory has demonstrated that mutations in the $S$. cerevisiae histone $\mathrm{H} 4$ amino terminus reduce transcriptional induction of $G A L I$ and $P H O 5$ promoters severely, but not $C U P I$ (62). Since the $G A L I$ and $P H O 5$ promoters are known to contain positioned nucleosomes which are displaced during gene activation $(63,64)$, perhaps a lack of nucleosomes in $\mathrm{UAS}_{\mathrm{CUP1}}$ accounts for the absence of a histone $\mathrm{H} 4$ deletion mutant phenotype with respect to CUPI transcription. A lack of nucleosomes in $\mathrm{UAS}_{\text {CUPI }}$ could allow the rapid binding of copper-activated ACE1, resulting in immediate activation of this important copper detoxification gene.

Once deposited at multiple sites within $\mathrm{UAS}_{\mathrm{CUP1}}$, how does ACE1 activate $C U P 1$ transcription? The production of a heterologous yeast DNA binding domain fused to the carboxy-terminal half of ACE1 indicates that this acidic region harbors much of the activation function of this MRTF (53). In vitro experiments have begun to shed light on the mode by which ACE1 activates transcription. Using a mouse extract for in vitro transcription reactions, both basal and copper-induced $C U P 1$ transcription was shown to require TATA element sequences (65). Although this region serves as a binding site for $S$. cerevisiae TFIID protein expressed in vitro, this preparation fostered basal, but not copperactivated ACE1 transcription in vitro. In contrast, TFIID partially purified from mouse or human cultured cells supported both basal and $\mathrm{Cu}-\mathrm{ACE} 1-$ mediated transcription in the mouse in vitro system.
These experiments were interpreted to suggest that recombinant TFIID lacks a modification or component required to productively interact with $A C E 1$ bound to $\mathrm{UAS}_{\mathrm{CUPI}}$. As has been suggested for a number of eukaryotic genes (66), the presence of a coactivator required for ACE1-activated $C U P 1$ transcription has been postulated (65). Berk's laboratory has demonstrated that yeast TFIID lacking an amino terminal region failed to activate $C U P 1$ transcription in vivo in response to $\mathrm{Cu}-\mathrm{ACE} 1$, however, basal transcription was unaltered (67). Perhaps this region of $S$. cerevisiae TFIID is important for direct or indirect interactions with ACE1, but not components of the basal transcription machinery. For which step of CUPI gene transcription is ACE1 required? Again, using a mouse cell extract for in vitro transcription reactions, it has been suggested that $A C E 1$ is required to form and maintain a stable preinitiation transcription complex (68). Further in vitro experiments using a homologous yeast in vitro transcription assay system should shed further light on the precise biochemical role ACE1 plays in CUPI transcription. An $S$. cerevisiae in vitro transcription system dependent upon $\mathrm{Cu}, \mathrm{A}$ $\mathrm{CE} 1$ and $\mathrm{UAS}_{\mathrm{CUPI}}$ has recently been developed and should facilitate such studies (G. Butler, M. Wootner, J. Jaehning and D. Thiele, unpublished). A working model for the copper-activated transcriptional induction of the $S$. cerevisiae CUPI gene is shown in Fig. 3.

\section{Metal-Responsive Transcription is Common in Eukaryotes}

Although the metallothionein genes have served as the major investigative model for metal-regulated gene transcription thus far, several other metal-responsive genes have been reported from such diverse organisms as algae, fungi, plants, rodents and humans.

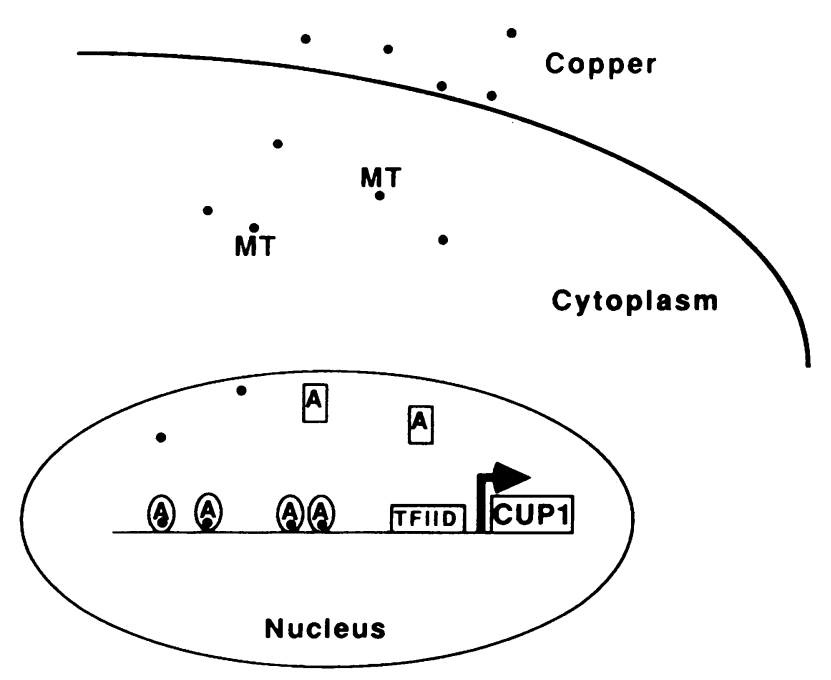

Figure 3. Model for Transcriptional Activation of the Saccharomyces cerevisiae $C U P I$ gene in response to copper. Copper $(\bullet)$ enters the yeast cell via an unknown transport mechanism and the fraction not bound by basal levels of metallothionein (MT) or other copper proteins enters the Nucleus. Copper is cooperatively bound in a polynuclear cluster arrangement to the constitutively synthesized, nuclear localized ACEl apoprotein ( $\Theta$ ). The metallation of ACE1 ( $Q$ ) effects a change in the tertiary structure of the amino-terminal DNA binding domain, thereby activating the ACEI DNA binding function. Once deposited at four sites within the $C U P I$ upstream activation sequences $\left(U_{A S} S_{C U P I}\right), C u-A C E 1$, in concert with Transcription Factor IID ( TFIID) and other unidentified factors, potently stimulates $C U P I$ transcription. Note that in cells lacking a $C U P I$ structural gene, the levels of intracellular free copper in untreated cells are elevated, thereby giving rise to high levels of $\mathrm{Cu}-\mathrm{ACE} 1$-mediated transcription in the absence of exogenous copper. 
What are these metal-responsive transcription units and what is known about the mechanisms for their metal-responsive transcription? Furthermore, what is the physiological significance of metal-responsive transcription with respect to these genes?

The algae Chlamydomonas reinhardtii presents an interesting and exciting model system for studies of metal-regulated gene transcription. In $C$. reinhardtii, the copper-containing plastocyanin

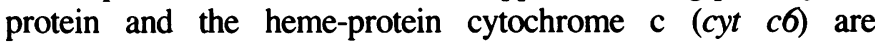
interchangably utilized as photosynthetic electron carriers. Interestingly, the accumulation of these proteins is reciprocally regulated by environmental copper levels, wherein, under conditions of copper sufficiency, plastocyanin accumulates due to an increase in the stability of the copper-metalloprotein. However, under low environmental copper levels, this protein turns over and the cyt c6 protein accumulates (69). A number of elegant experiments by Merchant and co-workers have recently demonstrated that the accumulation of cyt $c 6$ is due to transcriptional de-repression of the cyt $c 6$ gene, presumably involving a copper-sensing regulatory factor which either directly or indirectly influences cyt c6 gene transcription (70-72). The rapid kinetics of $c y t c 6$ gene derepression by copper are consistent with a pre-existing copper metalloregulatory factor, perhaps similar in principle to the rapid activation of the $S$. cerevisiae $C U P 1$ gene by constitutively synthesized ACE1 protein (46). In contrast to the characterized fungal metalloregulatory factors ACE1 and AMT1, however, $C$. reinhardtii cyt c6 gene repression is not responsive to $\mathrm{Ag}$, but is responsive, albeit to a lesser degree than copper, to elevated environmental $\mathrm{Hg}$ levels (71). Whether this is due to a reduced intracellular availability of $\mathrm{Hg}$ or a less efficient interaction of $\mathrm{Hg}$ with the same or a different metalloregulatory factor awaits the purification of this factor and the results of in vitro functional experiments. Interestingly, the metalloregulatory factors from $S$. cerevisiae and $C$. glabrata respond less efficiently to $\mathrm{Ag}$ than $\mathrm{Cu}$ for in vitro DNA binding and in vivo transcription activation, suggesting that $\mathrm{Cu}$ and $\mathrm{Ag}$ differentially interact with these metalloregulatory factors. The work on the $C$. reinhardtii cyt $c 6$ and plastocyanin gene systems underscores the intricate dynamics of metalloregulatory systems and the importance of understanding metal homeostasis for a complete comprehension of metal-regulated gene transcription. Little is currently known about cellular factors which accumulate, distribute and remove metals, nor if such factors are encoded by genes subject to metalregulated transcription.

Are there multiple target genes acted upon by the copper MRTF ACE1 in $S$. cerevisiae in response to changing environmental copper levels? Previous work demonstrated that the $S$. cerevisiae copper, zinc superoxide dismutase activity was low in cells grown in copper deficient medium, however, the addition of copper resulted in a 2-3 fold induction of $\mathrm{Cu}, \mathrm{Zn}$ SOD activity (73). These observations could be consistent with either post-translational incorporation of the essential copper into the metalloprotein, biosynthetic induction by copper, or both mechanisms. Kosman and colleagues (74) showed that both $\mathrm{Cu}, \mathrm{Zn}$ SOD protein and mRNA steady-state levels increase in response to exogenous $\mathrm{Cu}$, suggesting at least a biosynthetic response to elevated environmental $\mathrm{Cu}$ levels. Carri, et al demonstrated that a functional ACE1 gene was necessary for this effect (75). Recently, Gralla et al (76) have demonstrated that copper-activated ACE1 protein binds to a single site in the promoter of the $S$. cerevisiae $\mathrm{Cu}, \mathrm{Zn}$ SOD (SODI) gene. DNase I footprinting and methylation interferance studies have shown that the ACE1 binding site in the $S O D 1$ gene promoter is highly conserved in comparison to the four ACE1 binding sites in the CUPI promoter. In vivo expression analysis of an $S O D 1-l a c Z$ ( $\beta$-galactosidase) fusion gene bearing a non-functional ACE1 binding site clearly demonstrated that copper-activated ACE1 induces SODI transcription through this single binding site. Why should a copper-dependent DNA binding trans-activator activate transcription of the $\mathrm{Cu}, \mathrm{Zn}$ superoxide dismutase gene? Gralla et al (76) propose two physiologically relevant explanations for this observation. First, since $\mathrm{Cu}, \mathrm{Zn}$ SOD requires copper for activity, perhaps copper-charged ACE1 provides a signal that sufficient levels of intracellular copper are present to ensure the formation of active holoenzyme. Secondly, because copper stimulates the formation of highly toxic free radicals through redox interactions with $\mathrm{O}^{-}{ }_{2}(77)$, a substrate for SOD, perhaps SOD1 transcription is induced by Cu-ACE1 to lower the intracellular levels of reactive $\mathrm{O}^{-}$. It will be interesting to determine if other $S$. cerevisiae genes, possibly involved in diverse aspects of copper homeostasis, are transcriptionally activated or repressed by $\mathrm{Cu}-\mathrm{ACE} 1$.

In another lower eukaryote, the white rot basidiomycete Phanerochaete chrysosporium, mRNA levels from the manganese peroxidase gene (mnpl) have been shown to be dramatically elevated in the presence of manganese $(\mathrm{Mn})(78,79)$. This Mndependent induction of $\mathrm{mnpl}$ only occurs under conditions of nitrogen starvation and $\mathrm{Mn}$ is the only metal which has been found to mediate this effect. Interestingly, Gold and colleagues have found several regions of the mnpl promoter with strong homology to the mouse metallothionein MRE consensus (79). Since higher eukaryotic MT genes are known not to be inducible by $\mathrm{Mn}$, the mnpl gene could represent a related yet distinct metal-responsive system.

In soybean, two independent reports demonstrate that steadystate mRNA levels for the heat shock stress protein gene Gmhsp26-A (also calledG2-4) are induced by treatment of germinated soybean seeds with $\mathrm{CdCl}_{2}, \mathrm{Cu}$ or $\mathrm{Ag}(80,81)$. Transcription run-off experiments indicate that this effect is at the level of transcription and several other regions of this promoter region bear limited homology to the MRE sequences of higher eukaryotes. No studies concerning the mechanism for metalinducible transcription of this gene have been reported, however, the human (heat shock protein 70 gene) HSP70 gene has also been shown to be transcriptionally induced by a number of effectors which elicit a stress response, including heat, metals and amino acid analogs. All three conditions require HSP70 promoter sequences between -105 and -91 , although each of the induction conditions is strongly dependent on distinct basal level transcription elements within the HSP70 promoter, consisting of the TATA box, an AP1 site, SP1 site and CCAAT box elements (82). Although the sequence between -105 and -91 contains a functional heat shock element (HSE) and an overlapping region with some homology to MT gene MREs, a comprehensive mutagenic analysis of this region demonstrated that both heat- and metal-inducibility of HSP70 is mediated by the HSE (82). It is likely that, in this case, metals alter either the conformation of Heat Shock Transcription Factor (HSF) or its post translational modification, to allow a productive interaction with the HSE in HSP7O which results in the activation of $H S P 70$ transcription. Indeed, strong evidence hs been demonstrated for a role of both of these events in HSF activity $(83,84)$. Nonetheless, since metal-inducible HSP70 transcription is more dependent on basal level promoter elements than other forms of stress-activated transcription, metals may confer different types of conformational changes or posttranslational modification to HSF. 
Recently, Papaconstantinou and colleagues have demonstrated that two acute-phase protein genes from mouse, the serum $\alpha_{1}$-acid glycoprotein gene (AGP1) and the C-reactive protein gene (CRP), have elevated steady state levels of the corresponding mRNA species in response to treatment of mice with metals (85). The induction of these mRNA levels occurred in response to the following metals in order of decreasing potency: $\mathrm{Pb}, \mathrm{Cu}, \mathrm{Ni}, \mathrm{Zn}$, Mg. Furthermore, AGP mRNA levels were elevated in metal treated mice primarily in the liver, suggesting that perhaps one or more metal-responsive transcription factors may be controlled at the level of biosynthesis or activity in a tissue-specific manner. Transfection experiments with an AGP1 promoter-CAT (chloramphenicol acetyl transferase) fusion gene in HepG2 cells, a hepatoblastoma-derived cell line, demonstrated that this fusion is inducible by $\mathrm{HgCl}_{2}$, however, it remains to be established by nuclear run-on experiments whether these effects are exerted at the transcriptional or post-transcriptional level. Since this portion of the AGP1 promoter contains four regions with homology to the higher eukaryotic metallothionein MRE consensus sequence (86), perhaps these acute phase protein genes are transcriptionally responsive to the same metalloregulatory factors as MT genes. Interestingly, in both rat and mouse, the heme oxygenase gene (HO), which encodes an activity governing the rate-limiting step in heme catabolism, is transcriptionally induced in response to $\mathrm{CdCl}_{2}$ treatment (87). Both the dose-responsiveness and kinetics of $\mathrm{HO}$ gene transcription in response to $\mathrm{CdCl}_{2}$ are similar to that of rodent metallothionein genes, suggesting that $\mathrm{CdCl}_{2}$ may induce $\mathrm{HO}$ gene transcription by a similar mechanism. Consistent with these observations, the rat $\mathrm{HO}$ gene promoter contains two regions bearing close resemblance to $M T$ gene $M R E$ sequences, however, the role of these sequences in $\mathrm{CdCl}_{2}$-inducible $\mathrm{HO}$ gene transcription has not yet been reported.

Cadmium has been shown to induce steady state mRNA levels of two rat proto-oncogenes, $c$-myc approximately 30 -fold and $c$ jun 3-5 fold (88). Although the kinetics of induction of $c-m y c$ and $c$-jun in response to $\mathrm{CdCl}_{2}$ in rat $\mathrm{L671}$ myoblasts appears to be faster than for MT gene induction, maximal induction occurs at 5-10 $\mu \mathrm{M} \mathrm{CdCl}$, similar to that observed for MT gene induction. Whether the $c$-jun and $c$-myc gene promoters contain functional MRE-like elements has not yet been established, therefore, the mechanism for $\mathrm{CdCl}_{2}$-induced expression is currently unclear. The known carcinogenicity of cadmium (89), however, suggests that perhaps this property is mediated, at least in part, by hyper-expression of these proto-oncogene products. Furthermore, a recent report using Swiss 3T3 cells demonstrates the enhancement of $c$-fos mRNA levels in response to $\mathrm{Cd}$ or $\mathrm{Zn}$ administration independently of de novo protein synthesis (90).

The importance of metal-inducible transcription for analogous genes in different organisms has recently been shown to be drammatically different. The iron-storage protein ferritin is known to be biosynthetically controlled in rat, rabbit and human primarily at the translational level in response to environmental iron levels (91). Studies of the rat heavy $(\mathrm{H})$ and light $(\mathrm{L})$ chain ferritin subunit RNA have shown that, in addition, the $\mathrm{L}$ but not $\mathrm{H}$ mRNA species is also transcriptionally induced by iron approximately three-fold (92). In soybean cells, however, in vitro nuclear run-on assays have demonstrated that a soybean ferritin gene is transcriptionally induced approximately 45 -fold in response to iron administration (93). This transcriptional activation of the soybean ferritin gene would account for most, and perhaps all of the increase in ferritin protein observed under elevated environmental iron conditions. Although the biochemical mechanism for iron-induced ferritin gene transcription is currently unknown, perhaps an iron-responsive positive or negatively acting MRTF exists which plays a more dominant role in plants than in animal cells. This represents a very exciting area for future investigations into metal-regulated gene transcription and, additionally, provides an interesting model system for defining how organisms use both metal regulated transcription and translation to govern protein levels.

\section{SUMMARY}

This review has summarized many of the major aspects of metalregulated gene transcription in eukaryotic organisms as they are currently understood at the mechanistic level. Clearly, metals represent a class of important transcriptional effector molecules which regulate gene expression in different ways and both by activation or repression of gene transcription. To date, studies of metal-regulated transcription in fungi have resulted in the most detailed description of the structure, function and mechanisms of action of eukaryotic metal-responsive transcription factors. Recently, significant progress has been made in higher eukaryotic systems through the biochemical detection and purification of MRE binding proteins which may represent MRTFs. Additionally, perhaps fungi will be exploited for their genetics and ease of manipulation to clone and functionally analyze cDNAs for MRTFs from higher eukaryotes. The isolation of cDNAs for higher eukaryotic MRTFs will provide important tools for answering a number of interesting questions in metal-regulated gene transcription. How do higher eukaryotes activate MT gene transcription in response to a broad range of environmental metals? What are the tissue distributions of MRTFs and how does their activity correlate with the exposure of different tissues to varying concentrations of metals? What are the identities of other genes regulated by MRTFs and why are such genes metal-responsive? A comprehensive understanding of the detailed mechanisms for metal-regulated transcription will ultimately require an understanding of how eukaryotic cells sense, transport, distribute and remove metals from their environment. These questions provide an interesting and exciting area of investigation for geneticists, physiologists, molecular biologists, biophysicists and biochemists now and in the future.

\section{ACKNOWLEDGMENTS}

I wish to express my gratitude to the large number of investigators worldwide who have generously shared reprints, preprints and ideas during the formulation of this review. I am grateful to the members of my laboratory, G. Butler, K. Tamai, M. Szczypka, P. Zhou and T. Sosinowski, for their comments and helpful suggestions during the preparation of this review. Work in my laboratory on metal regulated gene expression is funded by the National Institutes of Health.

\section{REFERENCES}

1. Cotton, F.A. and Wilkinson, G. (1980) Advanced Inorganic Chemistry, A Comprehensive Text. John Wiley and Sons, New York.

2. Hom, N. (1984) In, Metabolism of Trace Metals in Man, Vol. II, (Rennert, O.M. and Chan, W.-Y., eds.) CRC Press, Boca Raton, Fla. pp. 26-52.

3. O'Halloran, T.V. (1989) in, Metal Ions in Biological Systems (Sigel, H. and Sigel, A., eds) Marcel Dekker, Inc. New York, pp. 105-146.

4. Kägi, J.H.R. and Schaffer, A. (1988) Biochemistry 27, 8509-8515.

5. Hamer, D. (1986) Ann. Rev. Biochem. 55, 913-951.

6. Durnam, D.M., Palmiter, R.D. (1981) J. Biol. Chem. 256, 5712-5716. 
7. Stuart, G.W., Searle, P.F., Chen, H.Y., Brinster, R.L. and Palmiter, R.D. (1984) Proc. Natl. Acad. Sci. USA 81, 7318-7322.

8. Carter, A.D., Felber, B.K., Walling, M.J., Jubier, M.-F., Schmidt, C.J. and Hamer, D.H. (1984) Proc. Natl. Acad. Sci. USA 81, 7392-7396

9. Karin, M., Haslinger, A., Heguy, A., Dietlin, T. and Cooke, T. (1987) Mol. Cell. Biol. 7, 606-613.

10. Otto, E., Allen, J.M., Young, J.E., Palmiter, R.D. and Maroni, G. (1987) Mol. Cell. Biol. 7, 1710-1715.

11. Harlow, P., Watkins, E., Thornton, R.D. and Nemer, M. (1989) Mol. Cell. Biol. 9, 5445-5455.

12. Andersen, R.D., Birren, B.W., Tapliz, S.J. and Herschman, H.R. (1986) Mol. Cell. Biol. 6, 302-314.

13. Zafarullah, M., Bonham, K. and Gedamu, L. (1988) Mol. Cell. Biol. 8, 4469-4476

14. Searle, P.F., Stuart, G.W. and Palmiter, R.D. (1987) In, Metallothionein II, Experientia Supplementation 52, 407-414, Birkhauser Verlag, Basal.

15. Cizewski Culotta, V. and Hamer, D.H. (1989) Mol. Cell Biol. 9, 1376-1380

16. Stuart, G.W., Searle, P.F. and Palmiter, R.D. (1985) Nature 317, 828-831.

17. Westin, G. and Schaffner, W. (1989) EMBO J. 7, 3763-3770.

18. Karin, M., Andersen, R.D., Slater, E., Smith, K. and Herschman, H.R. (1980) Nature 286, 295-297.

19. Séguin, C., Felber, B.K., Carter, A.D. and Hamer, D.H. (1984) Nature 312 , 781-785

20. Andersen, R.D., Taplitz, S.J., Wong, S., Bristol, G., Larkin, B. and Herschman, H.R. (1987). Mol. Cell. Biol. 7, 3574-3581.

21. Mueller, P.R., Salser, S.J. and Wold, B. (1988) Genes and Develop. 2, 412-427.

22. Andersen, R.D., Taplitz, S.J., Oberbauer, A.M., Calame, K.L. and Herschman, H.R. (1990) Nuc. Acids Res. 18, 6049-6055.

23. Kadonaga, J.T., Carner, K.R., Masiarz, F.R. and Tjian, R. (1987) Cell 51, $1079-1090$

24. Imbert J., Zafarullah, M., Cizewski Culotta, V., Gedamu, L. and Hamer, D. (1989) Mol. Cell. Biol. 9, 5315-5323.

25. Erdile, L.F., Heyer, W.-D., Kolodner, R. and Kelly, T.J. (1991) J. Biol. Chem. 266, 12090-12098.

26. Séguin, C. (1991) Gene 97, 295-300.

27. Labbè, S., Prévost, J., Remondelli, P., Leone, A. and Séguin, C. (1991) Nux. Acids Res. 19, 4225-4231.

28. Searle, P.F. (1990) Nuc. Acids Res. 18, 4683-4690.

29. Maguire, K.A., Webb, M.L., Garg, L.C. and Jacob, S.T. (1987) J. Biol. Chem. 209, 3932-3935.

30. Schöler, H.R. (1991) Trends in Genetics 7, 323-329.

31. Cousins, R.J. and Lee-Ambrose, L.M. (1992) J. Nutrition 122, 56-64.

32. Brenes-Pomales, A., Lindegren, G. and Lindegren, C.C. (1955) Nature 136, $841-842$.

33. Butt, T.R. and Ecker, D.J. (1987) Microbiol. Rev. 51, 351-364.

34. Hamer, D.H., Thiele, D.J. and Lemontt, J.E. (1985) Science 228, 685-690.

35. Ecker, D.J., Butt, T.R., Stermberg, E.J., Neeper, M.P., Debouck, C., Gorman, J.A. and Crooke, S.T. (1986) J. Biol. Chem. 261, 16895-16900.

36. Karin, M., Najarian, R., Haslinger, A., Valenzuela, P., Welch, J. and Fogel, S. (1984) Proc. Natl. Acad. Sci. USA 81, 337-341.

37. Butt, T.R., Sternberg, E.J., Gorman, J.A., Clark, P., Hamer, D., Rosenberg, M. and Crooke, S.T. (1984) Proc. Natl. Acad. Sci. USA 81, 3332-3336.

38. Winge, D.R., Nielson, K.B., Gray, W.R. and Hamer, D.H. (1985) J. Biol. Chem. 260, 14464-14470.

39. Fürst, P., Hu, S., Hackett, R. and Hamer, D. (1988) Cell 55, 705-717.

40. Jeyaprakash, A., Welch, J.W. and Fogel, S. (1991) Mol. Gen. Genet. 225 , 363-368.

41. Münger, K., Germann, U.A. and Lerch, K. (1987) J. Biol. Chem. 262, 7363-7367.

42. Thiele, D.J. and Hamer, D.H. (1986) Mol. Cell. Biol. 6, 1158-1163.

43. Thiele, D.J. (1988) Mol. Cell. Biol. 8, 2745-2752.

44. Welch, J., Fogel, S., Buchman, C. and Karin, M. (1989) EMBO J. 8, 255-260.

45. Buchman, C., Skroch, P., Dixon, W., Tullius, T.D. and Karin, M. (1990) Mol. Cell. Biol. 10, 4778-4787.

46. Szczypka, M.S. and Thiele, D.J. (1989) Mol. Cell. Biol. 9, 421-429.

47. Butler, G. and Thiele, D.J. (1991) Mol. Cell. Biol. 11, 476-485.

48. Mehra, R.K., Garey, J.R., Butt, T.R., Gray, W.R. and Winge, D.R. (1989) J. Biol. Chem. 264, 19747-19753.

49. Mehra, R.K., Garey, J.R. and Winge, D.R. (1990) J. Biol. Chem. 265, 6369-6375

50. Zhou, P. and Thiele, D.J. (1991) Proc. Natl. Acad. Sci. USA 88, 6112-6116.

51. Mehra, R.K. and Winge, D.R. (1991) J. Cell. Biochem. 45, 30-40.

52. Barns, S.M., Lane, D.J., Sogin, M.L., Bibeau, C. and Weisburg, W.G. (1991) J. Bacteriol. 173, 2250-2255.
53. Hu, S., Furst, P. and Hamer, D. (1990). New Biol. 2, 1-13.

54. Nakagawa, K.H., Inouye, C., Hedman, B., Karin, M., Tullius, T.D. and Hodgson, K.O. (1991) J. Am. Chem. Soc. 113, 3621-3623.

55. Dameron, C.T., Winge, D.R., George, G.N., Sansone, M., Hu, S. and Hamer, D. (1991) Proc. Natl. Acad. Sci. USA 88, 6127-6131.

56. Fürst, P. and Hamer, D. (1989) Proc. Natl. Acad. Sci. USA 86, 5267-5271.

57. Thiele, D.J., Walling, M.J. and Hamer, D.H. (1986) Science 231, 854-856.

58. Wright, C.F., Hamer, D.H. and McKenney, K. (1988) J. Biol. Chem. 263 $1570-1574$.

59. Huibregtse, J.M., Engelke, D.R. and Thiele, D.J. (1989) Proc. Natl. Acad. Sci. USA 86, 65-69.

60. Evans, C.F., Engelke, D.R. and Thiele, D.J. (1990) Mol. Cell. Biol. 10, 426-429.

61. Grunstein, M. (1990) Trends Genet. 6, 395-400.

62. Durrin, L.K., Mann, R.K., Kayne, P.S. and Grunstein, M. (1991) Cell 65, 1023-1031.

63. Han, M. and Grunstein, M. (1988) Cell 55, 1137-1145.

64. Almer, A., Rudolph, H., Hinnen, A. and Horz, W. (1986) EMBO J 5 , $2689-2696$

65. Kambadur, R., Culotta, V. and Hamer, D. (1990) Proc. Natl. Acad. Sci. USA 87, 9168-9172.

66. Stanway, C.A. (1991) Bio Essays 13, 241-242.

67. Zhou, Q., Schmidt, M.C. and Berk, A.J. (1991) EMBO J 10, 1843-1852.

68. Cizewski Culotta, V., Hsu, T., Hu, S., Fürst, P. and Hamer, D.H. (1989) Proc. Natl. Acad. Sci. USA 86, 8377-8381.

69. Merchant, S. and Bogorad, L. (1986) Mol. Cell. Biol. 6, 462-469.

70. Merchant, S. and Bogorad, L. (1987) J. Biol. Chem. 262, 9062-9067.

71. Hill, K.L., Li, H.H., Singer, J. and Merchant S. (1991) J. Biol. Chem. 266, 15060-15067.

72. Merchant, S., Hill, K. and Howe, G. (1991) EMBO J. 10, 1383-1389.

73. Gregory, E.M., Goscin, S.A. and Fridovich, I. (1974) J. Bacteriol. 117, 456-460.

74. Greco, M.A., Hrab, D.I., Magner, W. and Kosman, D.J. (1990) J. Bacteriol. 172, 317-325

75. Carri, M.T., Galiazzo, F., Ciriolo, M.R. and Rotilio, G. (1991) FEBS Lett. 2278, 263-266.

76. Gralla, E.B., Thiele, D.J., Silar, P. and Valentine, J.S. (1991) Proc. Natl. Acad. Sci. USA 88, 8558-8562.

77. Halliwell, B. and Gutteridge, J.M.C. (1984) Biochem. J. 219, 1-14.

78. Brown, J.A., Glenn, J.K. and Gold, M.H. (1990) J. Bacteriol. 172 $3125-3130$.

79. Brown, J.A., Alic, M. and Gold, M.H. (1991) J. Bacteriol. 173, 4101-4106.

80. Czarnecka, E., Nagao, R.T., Key, J.L. and Gurley, W.B. (1988) Mol. Cell. Biol. 8, 1113-1122.

81. Hagan, G., Uhrhammer, N. and Guilfoyle, T.J. (1988) J. Biol. Chem. 263 , 6442-6446.

82. Williams, G.T. and Morimoto, R.I. (1990) Mol. Cell. Biol. 10, 3125-3136.

83. Zimarino, V., Wilson, S. and Wu, C. (1990) Science 249, 546-549.

84. Laron, J.S., Schuetz, T.J. and Kingston, R.E. (1988) Nature 335, 372-375.

85. Yiangou, M., Ge, X., Carter, K.C. and Papaconstaninou, J. (1991) Biochemistry 30, 3798-3806.

86. Cooper, R., Eckley, D.M. and Papaconstantinou, J. (1987) Biochemistry 26, $5244-5250$.

87. Alam, J., Shibahara, S. and Smith, A. (1989) J. Biol. Chem. 264, 6371-6375.

88. Jin, P. and Ringertz, N.R. (1990) J. Biol. Chem. 265, 14061-14064

89. Leonard, A., Gerber, G.B., Jacquet, P. and Lquwerys, R.R. (1984) in Mutagenicity, Carcinogenicity and Teratogenicity of Industrial Pullutants (KirchVolder, M., ed.) pp. 59-125, Plenum Publishing Corp., New York.

90. Epner, D.E. and Herschman, H.R. (1991) J. Cell Physiol. 148, 68-74.

91. Theil, E. (1987) Ann. Rev. Biochem. 56, 289-315.

92. White, K. and Munro, H. (1988) J. Biol. Chem. 263, 8938-8942.

93. Lescure, A.-M., Proudhon, O., Pesey, H., Ragland, M., Theil, E.C. and Briat, J.-F. (1991) Proc. Natl. Acad. Sci. USA 88, 8222-8226. 